(C) [2009] IEEE. Reprinted, with permission, from Xu, Wei; Zhu, Jianguo; Guo, Youguang; Wang, Yi; Zhang, Yongchang; Tan, Longcheng. Equivalent Circuits for Singled-sided Linear Induction Motors', Proceedings of IEEE Energy Conversion Conference \& Expo, pp. 1288-1295. This material is posted here with permission of the IEEE. Such permission of the IEEE does not in any way imply IEEE endorsement of any of the University of Technology, Sydney's products or services. Internal or personal use of this material is permitted. However, permission to reprint/republish this material for advertising or promotional purposes or for creating new collective works for resale or redistribution must be obtained from the IEEE by writing to pubs-permissions@ieee.org. By choosing to view this document, you agree to all provisions of the copyright laws protecting it. 


\section{Equivalent Circuits for Single-sided Linear Induction Motors}

\author{
Wei Xu, Jianguo Zhu, Youguang Guo, \\ Yi Wang, Yongchang Zhang \\ School of Electrical, Mechanical and Mechatronic Systems \\ University of Technology Sydney \\ Sydney, Australia \\ wxu@eng.uts.edu.au, joe@eng.uts.edu.au
}

\begin{abstract}
Single-sided linear induction motors (SLIMs) have lately been applied in transportation system traction drives, especially in the intermediate speed range. They have merits such as the ability to exert thrust on the secondary without mechanical contact, high acceleration or deceleration, less wheel wear, small turning circle radius and flexible road line. The theory of operation for these machines can be directly derived from rotary induction motors, but several issues involving the transversal edge and the longitudinal end effects, and half-filled slots at the primary ends, need to be investigated. In this paper, a $\mathbf{T}$ model equivalent circuit is proposed which is based on onedimensional magnetic equations of the air-gap, where half-filled slots are considered by an equivalent pole number. Then, it deduces two-axis equivalent circuits to study the SLIM dynamic performance. The theoretical analyses have been validated by experimental results on SLIM prototypes.
\end{abstract}

Index Terms-Single-sided linear induction motor (SLIM), transversal edge effect, longitudinal end effect, equivalent circuit, correct coefficient, mutual inductance, secondary resistance, steady performance, dynamic performance, control scheme.

\section{NOMENCLATURE}

$\varepsilon \quad$ Primary winding short pitch

$\omega_{e} \quad$ Angular frequency of power supply

$\tau \quad$ Pole pitch

$d \quad$ Secondary sheet thickness

$f_{\mathrm{s}} \quad$ Primary frequency

$g_{\mathrm{e}} \quad$ Equivalent air gap length

$g_{\mathrm{m}} \quad$ Mechanical air gap length

$l_{\delta} \quad$ Primary lamination width

$p_{\mathrm{e}} \quad$ Equivalent number of pole pairs

$C_{\mathrm{r}}(\mathrm{s})$ Transversal edge effect coefficient for the secondary resistance

$C_{\mathrm{x}}(\mathrm{s})$ Transversal edge effect coefficient for the mutual inductance

$K_{\mathrm{r}}(\mathrm{s})$ Longitudinal end effect coefficient for secondary resistance

$K_{\mathrm{x}}(\mathrm{s})$ Longitudinal end effect coefficient for mutual inductance

$L_{\mathrm{m}} \quad$ Mutual inductance in two-axis circuit

$L_{\mathrm{m} 1} \quad$ Mutual inductance in per-phase circuit

$L_{\mathrm{ls}} \quad$ Primary leakage inductance

$L_{\mathrm{Ir}} \quad$ Secondary leakage inductance

$R_{\mathrm{r}} \quad$ Secondary resistance

\section{INTRODUCTION}

The structure diagram of a single-sided linear induction motor (SLIM) is shown in Fig. 1. The SLIM's primary side can be simply regarded as a rotary induction motor (RIM) stator which is cut-open and rolled flat. The secondary side is similar to the RIM rotor which often consists of a sheet conductor, such as copper or aluminum, with a solid back iron acting as the return path of the magnetic flux. The thrust

\author{
Longcheng Tan \\ Institute of Electrical Engineering \\ Chinese Academy of Sciences \\ Beijing, China \\ lchtan@mail.iee.ac.cn
}

corresponding to the RIM torque can be produced by the reaction between the air gap flux density and the eddy current in the secondary sheet.

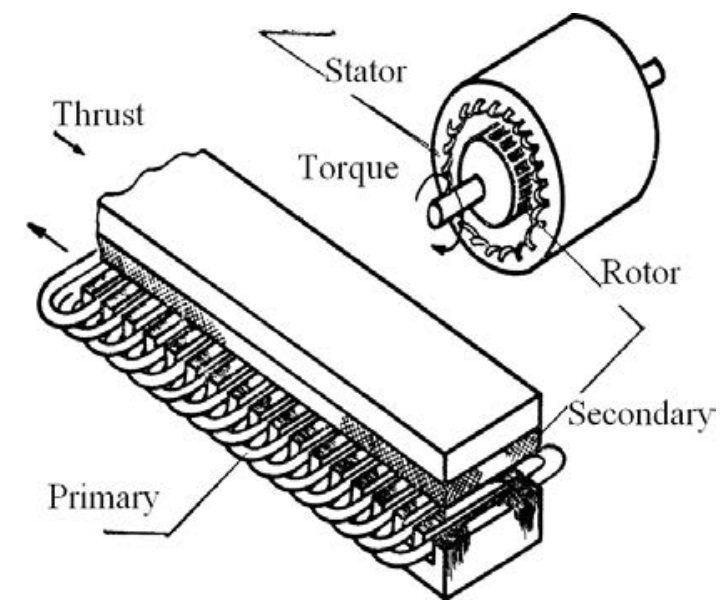

Fig. 1. Structure of an SLIM.

A train driven by SLIM, also called as linear metro, has been paid attention by academia and industry for more than two decades. By now, there are more than twenty commercial linear metro lines of about 400 kilometers in the world, such as the Kennedy air line in America, the linear metro in Japan, the Vancouver light train in Canada, the Guangzhou subway line 4 and the Beijing airport rapid transport line in China. The typical SLIM drive structure and system diagrams are illustrated in Fig. 2. It can be seen that the SLIM primary is hanged below the redirector, which is supplied by the inverter on the vehicle. The secondary is flatted on the railway track, which consists of a $5 \mathrm{~mm}$ thick copper/aluminum conductance fleet and a $20 \mathrm{~mm}$ thick back iron. When the primary three-phase windings are input with AC current, they can build up air gap flux linkage and induce eddy current in the secondary sheet. This eddy current will react with aforementioned air gap flux linkage so as to produce horizontal electromagnetic thrust that can drive the vehicle forward directly without friction between wheel and track.

The SLIM's special structure means that its performance is a little different from that of an RIM. As we know, in the RIM, an accurate equivalent circuit model can be derived easily based on the symmetrical geometry per pole. Unfortunately, it is not as straightforward to gain the equivalent circuit for an SLIM mainly for the following three 
causes.

(1) As the SLIM primary moves, a new flux is continuously developed at the primary entrance side, while the air gap flux disappears quickly at the exit side. By the influence of the sudden generation and disappearance of the air gap penetrating flux density, an amount of eddy current in anti-direction to the primary current will occur in the secondary sheet, which correspondingly affects the air gap flux profile along the longitudinal direction (x-axis) as illustrated in Fig. 3. This phenomenon is called "longitudinal end effect", which could increase the copper loss, and decrease the mutual inductance as the velocity goes up. In the end, the effective electromagnetic thrust will be reduced because of its attenuated air gap average flux linkage [5].

(2) The different width between primary lamination and secondary sheet can result in non-uniform flux density, which may increase the secondary equivalent resistance. The phenomenon is named as "transversal edge effect".

(3) For the cut-open primary magnetic circuit, there exist half-filled slots in the primary ends. Hence, three-phase magnetic circuits are not symmetrical with each other. Threephase currents are not symmetrical even when excited by three-phase balanced voltages. The half-filled slots will affect the air gap flux density distribution so as to result in some alteration in mutual inductance, leakage inductance, and secondary equivalent resistance.

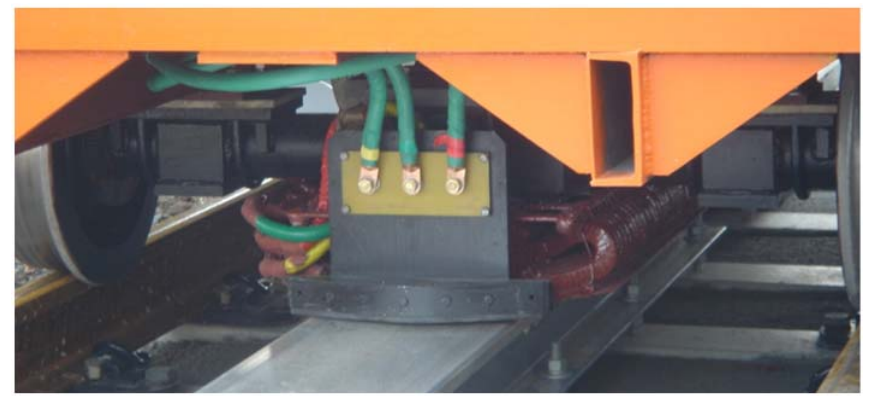

(a)

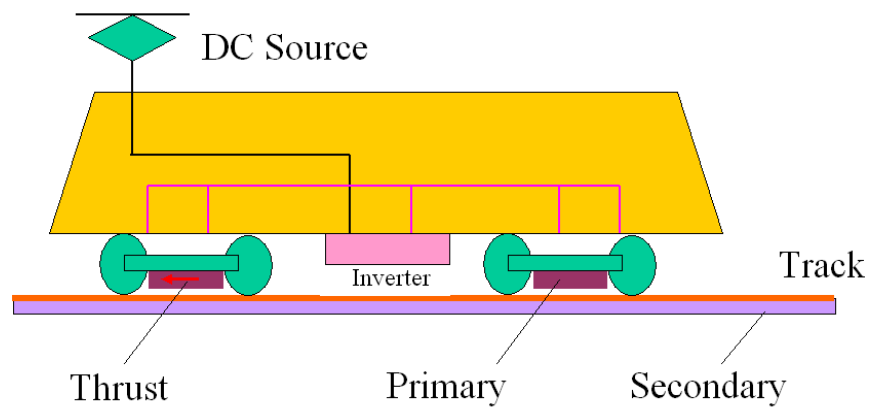

(b)

Fig. 2. Simple vehicle system diagrams propelled by the SLIM: (a) photo of an SLIM driven vehicle, (b) diagram of the drive system.
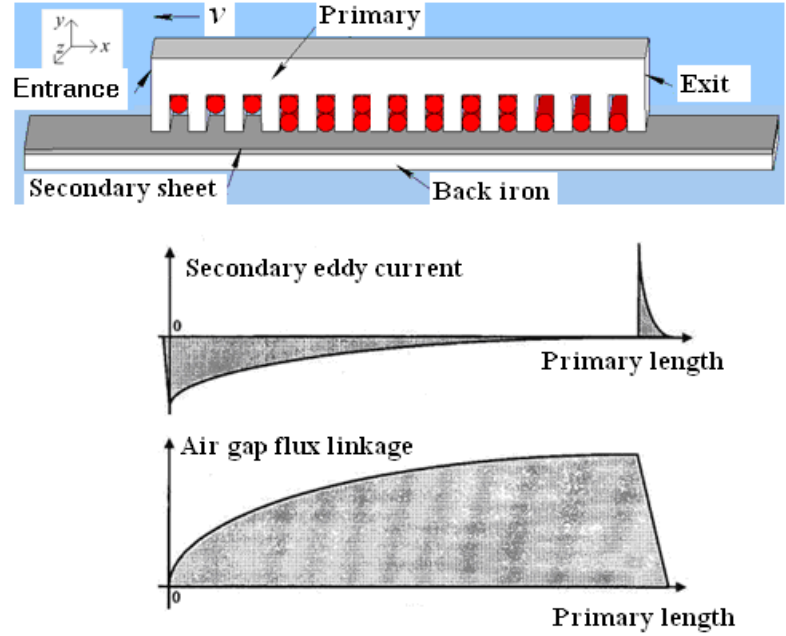

Fig. 3. Profiles of secondary eddy current and air gap flux linkage.

During the past several decades, plenty of papers have been available on the SLIM performance analysis, involving steady and dynamic states. Ref. [1] based on the RIM Tmodel equivalent circuit provided one simple and useful force function expression according to the secondary eddy current average value by energy conversion balance theorem. This function is affected by the SLIM speed, secondary resistance, secondary inductance, and some structural parameters, such as the primary length. The per-phase simplified model can be used to predict the SLIM output thrust and vertical force conveniently.

Refs. [2] and [3] according to the results in [1] deduced the two-axis models ( $d q$ or $\alpha \beta$ ), which can be applied in vector control or direct torque control to predict the dynamic performance. However, the derivation process of the revised force function is very coarse on the assumption that eddy current in the secondary sheet decreases from maximum to zero by exponential attenuation only in the primary length range. It only considers the mutual inductance influenced by the eddy current, disregarding its effect on the secondary resistance. The analytic results, such as the mutual variance, are with increasing error with the measurement as the velocity goes up.

Ref. [4] derived an equivalent circuit model from the poleby-pole method based on winding functions of the primary winding. One tenth order set of differential equations are derived to describe a basic model for the four pole machine, and higher order system of equations can be provided as the pole number goes up.

Ref. [5] based on the winding function method divided the SLIM air gap flux density into three components, i.e. primary fundamental, secondary fundamental, and secondary eddy current parts. Then it derived these three-group function expressions and further achieved inductance, secondary resistance and other parameters so as to analyze the SLIM performance. The winding function method can study the steady, transient, and dynamic performance. However, the expressions of secondary winding function distributions are 
achieved by some hypothesis.

Field theory was utilized in developing the lumped parameter LIM model [6], in which end effect, field diffusion in the secondary sheet, skin effect and back-iron saturation were considered. However, the resultant lumped-parameter models look very complicated for practical use of modeling and control.

Gieras and Faiz developed an equivalent circuit by superposing the synchronous wave and the pulsating wave caused by the end effect [7]-[8]. More experiments should be made to verify the simulation investigation. Several different models were developed from the electromagnetic relation in the air gap through a Fourier-series approach [9]-[11]. However, it requires more substantial computing time to gain some useful data, which accuracy depends closely on many initially given parameters. If some key values are not initialized rationally, the final solution cannot be achieved due to non-convergence.

This paper presents the performance analysis of two SLIMs by using a per-phase T-model equivalent circuit derived from one dimensional flux density equations [12]. Among the five major parameters involving primary resistance, primary leakage inductance, mutual inductance, secondary resistance, and secondary inductance, the mutual inductance and secondary resistance are influenced by the edge and end effects greatly which can be corrected by four relative coefficients, i.e. $K_{\mathrm{r}}(\mathrm{s}), K_{\mathrm{x}}(\mathrm{s}), C_{\mathrm{r}}(\mathrm{s})$ and $C_{\mathrm{x}}(\mathrm{s})$. Moreover, two-axis equivalent circuits ( $d q$ or $\alpha \beta$ ) according to the $\mathrm{T}$ model equivalent circuit are obtained using a flux linkage conservation method, which are analogous to those of the RIM in a two-axis coordination system. The LIM dynamic parameters, especially the mutual inductance and secondary resistance, can be analyzed by using the four coefficients. Experimental verification indicates that both the $\mathrm{T}$ model and the new two-axis circuits are reasonable for describing the steady and dynamic performance of the SLIM. These two models can provide good guidance for the electromagnetic design and control scheme implementation for SLIM.

\section{T-Model Equivalent CiRCUIT}

Fig. 4 shows the SLIM longitudinal side view, transversal side view, and one dimensional analytical model. In terms of one-dimensional analysis we can calculate the phase currents and excitation voltages. The air-gap flux linkage can be obtained using Maxwell field equations and solved using the complex power method with a conformal transformation which considers the effects of half-filled slots, yoke magnetic saturation and back iron resistance. Using the equal complex power relationship between magnetic field and electric circuit, we can obtain several circuit parameters, such as mutual inductance $L_{\mathrm{m} 1}$, secondary resistance $R_{\mathrm{r}}$, primary leakage inductance $L_{\mathrm{ls}}$, secondary leakage inductance $L_{\mathrm{l}}$, longitudinal end effect coefficients $K_{\mathrm{r}}(\mathrm{s})$ and $K_{\mathrm{x}}(\mathrm{s})$, and transverse edge effect coefficients $C_{\mathrm{r}}(\mathrm{s})$ and $C_{\mathrm{x}}(\mathrm{s})$. Their comprehensive derivation can be found in [12] and [13]. The T-model equivalent circuit is shown in Fig. 5.

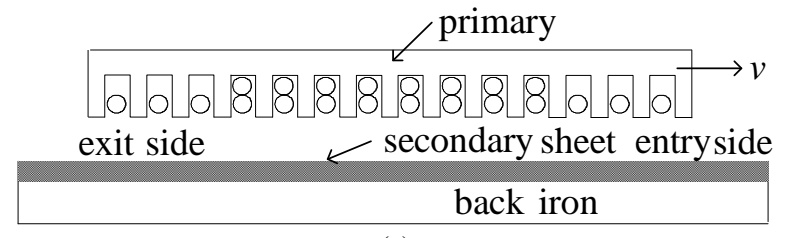

(a)

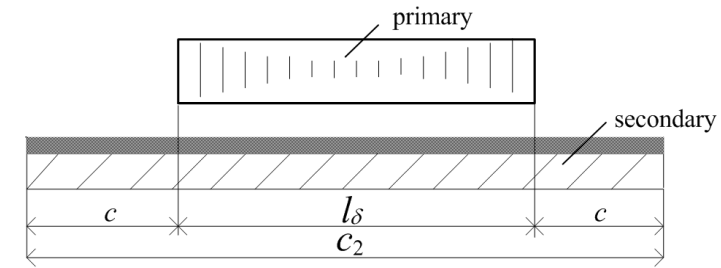

(b)

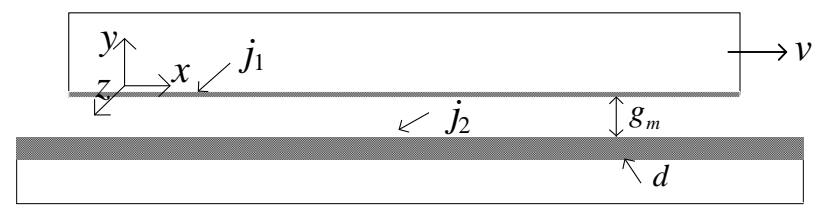

(c)

Fig. 4. Structure and one-dimensional analytical model of SLIM: (a) Longitudinal side view, (b) Transversal side view, (c) Analysis model.

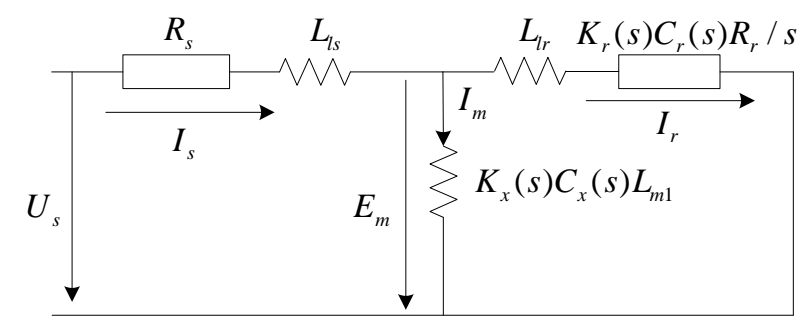

Fig. 5. T-model equivalent circuit of SLIM.

The longitudinal end-effect coefficients $K_{\mathrm{r}}(\mathrm{s})$ and $K_{\mathrm{x}}(\mathrm{s})$ are denoted by

$$
\begin{aligned}
& K_{r}(s)=\frac{s G}{2 p_{e} \tau \sqrt{1+(S G)^{2}}} \frac{C_{1}^{2}+C_{2}^{2}}{C_{1}} \\
& K_{x}(s)=\frac{1}{2 p_{e} \tau \sqrt{1+(S G)^{2}}} \frac{C_{1}^{2}+C_{2}^{2}}{C_{2}}
\end{aligned}
$$

where $C_{1}$ and $C_{2}$ are the functions of slip $s$ and goodness factor $G, \tau$ is the primary pole pitch, $p_{\mathrm{e}}$ is the number of equivalent pole pairs calculated by

$$
p_{e}=\frac{(2 p-1)^{2}}{4 p-3+\varepsilon /\left(m_{1} q\right)}
$$

where $p$ is the actual number of pole pairs, $m_{1}$ is the primary phase number, $q$ is the number of coil sides per phase per pole, and $\varepsilon$ is the length of short pitch.

The transversal end-effect coefficients $C_{\mathrm{r}}(\mathrm{s})$ and $C_{\mathrm{x}}(\mathrm{s})$ are given by 


$$
\begin{aligned}
C_{r}(s) & =\frac{s G\left[\mathrm{R}_{e}^{2}[T]+\mathrm{I}_{m}^{2}[T]\right]}{\mathrm{R}_{\mathrm{e}}[T]} \\
C_{x}(s) & =\frac{\mathrm{R}_{e}^{2}[T]+\mathrm{I}_{m}^{2}[T]}{\mathrm{I}_{\mathrm{m}}[T]}
\end{aligned}
$$

where $T$ is the function of slip, goodness factor and motor structure parameters, and $\mathrm{R}_{\mathrm{e}}$ and $\mathrm{I}_{\mathrm{m}}$ are the real and image parts of the complex $T$, respectively. Here $T$ is expressed by

$$
T=j\left[\gamma^{2}+\left(1-\gamma^{2}\right) \frac{\lambda}{0.5 l_{\delta} \alpha} \operatorname{th}\left(0.5 l_{\delta} \alpha\right)\right]
$$

where $l_{\delta}$ is half the primary lamination width, $\alpha$ is the ratio of $c$ to $\tau$, and $c$ is half the width of the secondary sheet overhang indicated in Fig. 4(b). $\gamma$ and $\lambda$ can be obtained by

$$
\begin{gathered}
\lambda=\frac{1}{1+\frac{1}{\gamma} \operatorname{th}\left(0.5 l_{\delta} \alpha\right) \operatorname{th}\left[0.5 K\left(c_{2}-l_{\delta}\right)\right]} \\
\gamma^{2}=\frac{1}{1+j s G}
\end{gathered}
$$

where $K$ is the function of slip and motor structure parameters, and $c_{2}$ is the width of the secondary sheet.

The five parameters in the T-circuit can be calculated as below. The primary resistance $r_{1}$ is

$$
r_{1}=\rho_{c u} \times 2 l_{a v} W_{1} / S_{c u}
$$

where $\rho_{C u}$ is the resistivity of copper, $l_{a v}$ is half the average length of the primary winding loop, $W_{1}$ is the number of turns of the primary per phase in series, and $S_{c u}$ is the effective cross-sectional area of the primary winding conductor.

The primary leakage inductance $L_{\mathrm{ls}}$ is

$$
L_{l s}=0.025 W_{1}^{2} \frac{l_{\delta}}{q}\left(\frac{\lambda_{s}}{p}+\frac{\lambda_{t}+\lambda_{e}+\lambda_{d}}{p_{e}}\right)
$$

where $\lambda_{\mathrm{s}}$ is the primary slot leakage magnetic conductance, $\lambda_{\mathrm{t}}$ the primary tooth leakage magnetic conductance, $\lambda_{\mathrm{e}}$ the primary winding end leakage magnetic conductance, and $\lambda_{\mathrm{d}}$ the primary harmonic leakage magnetic conductance.

The secondary resistance is composed of those of conducting sheet and back iron because the flux can penetrate through the aluminum or copper sheet [13], and then enter the back iron. The depth of flux density into back iron $d_{F e}$ is

$$
d_{F e}=\sqrt{\frac{2 \rho_{F e}}{s \omega_{e} \mu_{F e}}}
$$

where $\rho_{\mathrm{Fe}}$ is back iron resistivity, $\mu_{\mathrm{Fe}}$ the permeability of back iron, and $\omega_{\mathrm{e}}$ the primary synchronous angular frequency. The resistance of secondary conducting sheet $r_{2 \text { sheet }}$ is

$$
r_{\text {2sheet }}=4 m_{1} \rho_{\text {sheet }} \frac{\left(W_{1} K_{W 1}\right)^{2}}{2 p_{e}} \frac{l_{\delta}}{d \tau}
$$

where $\rho_{\text {sheet }}$ is the resistivity of the secondary conduction sheet, and $K_{\mathrm{w} 1}$ is primary winding coefficient.

The resistance of secondary back iron $r_{2 \mathrm{Fe}}$ is

$$
r_{2 F e}=4 m_{1} \rho_{F e} \frac{\left(W_{1} K_{W 1}\right)^{2}}{2 p_{e}} \frac{l_{\delta}}{d_{F e} \tau}
$$

Therefore, the secondary equivalent resistance $r_{2}$ is

$$
r_{2}=\frac{r_{2 \text { sheet }} r_{2 \mathrm{Fe}}}{r_{\text {2sheet }}+r_{2 \mathrm{Fe}}}
$$

The secondary leakage reactance is

$$
L_{l r}=\frac{r_{2}}{2 \pi f_{s} s} B_{1} \operatorname{sh}(2 K d)
$$

where $f_{\mathrm{s}}$ is the primary frequency, $B_{1}$ is the function of slip, primary frequency, and machine structure parameters.

The exciting reaction is

$$
L_{m}=4 m_{1} \mu_{0}\left(W_{1} K_{W_{1}}\right)^{2} \frac{l_{\delta} V_{s}}{4 \pi^{2} f_{s} g_{e} p_{e}}
$$

where $V_{\mathrm{s}}$ is the synchronous velocity of primary side, and $g_{\mathrm{e}}$ is the equivalent air gap length.

After the approximate regulation by the four coefficients and the equivalent number of pole pairs, the SLIM T model equivalent circuit indicated in Fig. 5 is similar with that of the RIM. The influence by longitudinal and transversal end effects and half-filled slots can be estimated by the corresponding coefficients. The revised SLIM model based on the equivalent pole pairs can be regarded as three-phase magnetic circuit symmetry. When $K_{\mathrm{r}}(\mathrm{s})=K_{\mathrm{x}}(\mathrm{s})$ $=C_{\mathrm{r}}(\mathrm{s})=C_{\mathrm{x}}(\mathrm{s})=1$, i.e., when the longitudinal and transverse effects are neglected, the circuit can be simplified as the same as that of RIM. Therefore, it is very convenient to analyze the performance of SLIM in the similar way as that of RIM based on the circuit [12]-[16].

\section{VERIFICATION OF T-MOdEL EquiVAlENT CiRCUIT}

In order to validate the T-model equivalent circuit in Fig. 5, lots of steady state performance analysis has been made in [13]. Here two kinds of SLIM performance evaluations are given out, i.e. the 12000 Japanese SLIM and an arc SLIM. Their main dimensions are shown in Table I.

TABLE I

MAIN DIMENSIONS OF TWO SLIMS

\begin{tabular}{lll}
\hline Items & 12000 & Arc \\
Pole pitch (m) & 0.2808 & 0.117 \\
Pole pairs & 4 & 3 \\
Primary length/width(m) & $2.476 / 0.3$ & $0.80 / 0.1$ \\
Secondary width(m) & 0.36 & 0.17 \\
Secondary sheet thickness (mm) & 7 & 5 \\
Air gap length (mm) & 9 & 10 \\
Slot number & 79 & 41 \\
\hline
\end{tabular}

Fig. 6 shows the 12000 SLIM performance curves, including thrust, slip frequency, power factor, efficiency, and current [11]. The base speed is $40 \mathrm{~km} / \mathrm{h}$. There are two working models in the operating regions, i.e. "constant 
current” below the base speed and "constant voltage” beyond the base. It can be seen that the simulation results about the power factor, efficiency, and phase current are approximately in harmony with the measurements in [11].

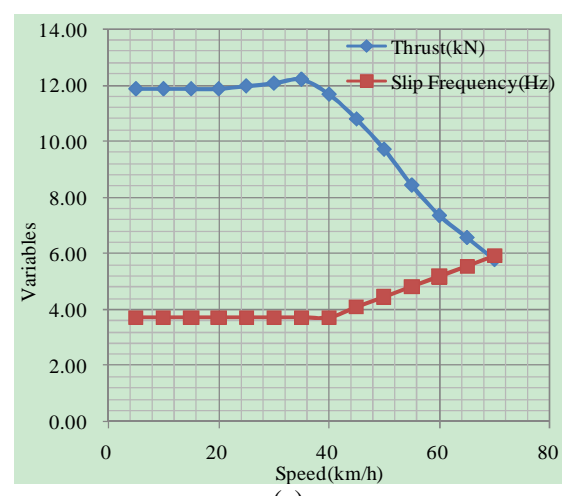

(a)

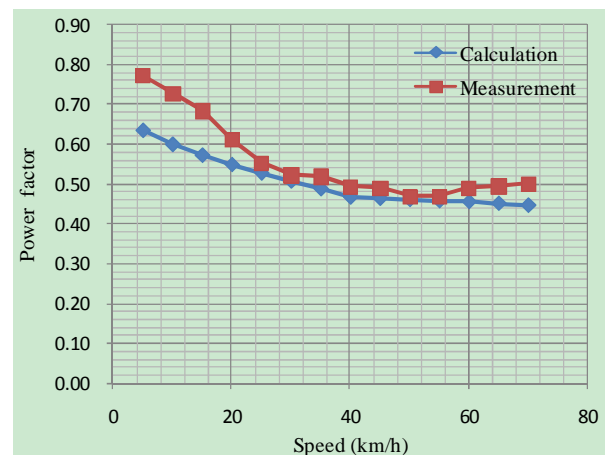

(b)

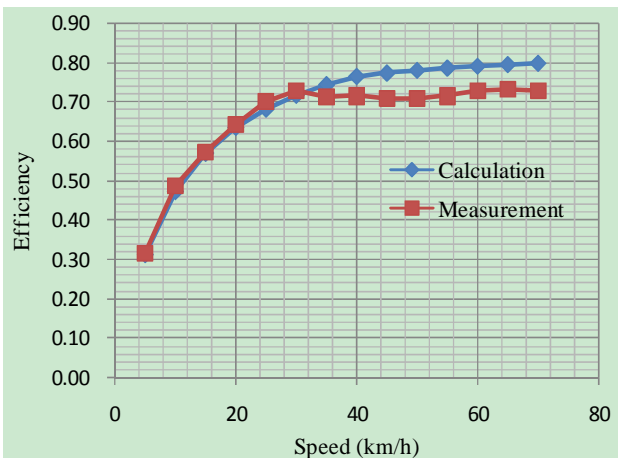

(c)

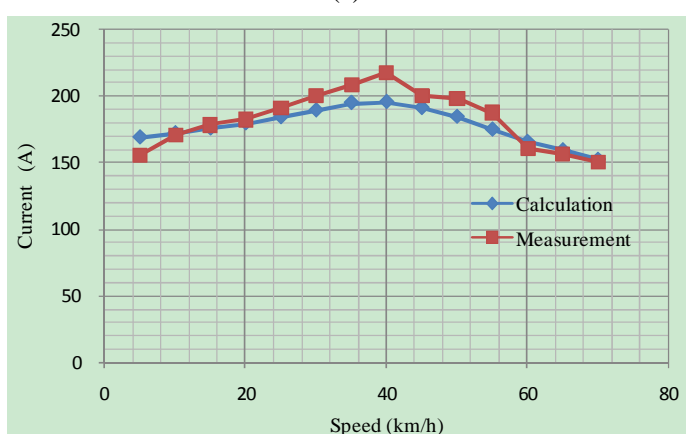

(d)

Fig. 6. Steady performance of Japanese 12000 SLIM [11]: (a) Thrust and slip frequency; (b) Power factor; (c) Efficiency; and (d) Phase current.
Fig. 7 shows the arc SLIM prototype experimental bench. It has a rotor which is formed on the rim of the large-radius flywheel whose primary is fed by a converter. The load cell is a DC machine which is connected to the shaft of the SLIM rig by belts. The DC machine can operate at any desired speed and load below the rating values to provide different operational points. The measurement sensors located between SLIM and DC load can record the SLIM velocity, load power and thrust.

Fig. 8 plots the thrust curves in the constant current constant frequency mode or the constant voltage constant frequency mode. It can be seen clearly that the thrust is reduced due to the end effect, especially in the high speed region. Moreover, the thrust excited by the constant voltage mode decreases much faster than the constant current mode because of its faster air gap flux attenuation. The thrust simulation agrees with the measurements reasonably in various velocities.

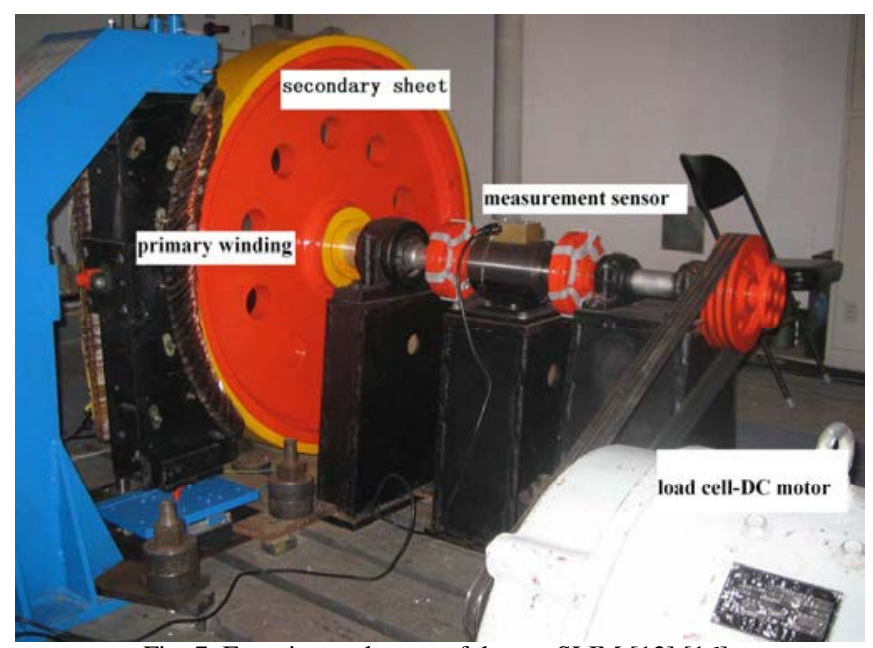

Fig. 7. Experimental setup of the arc SLIM [12] [16].

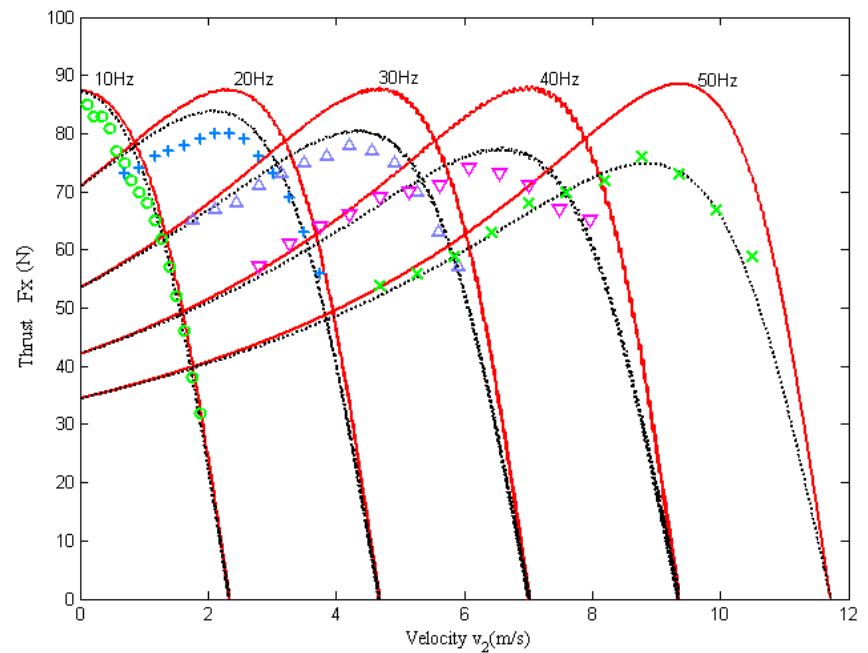

(a) 


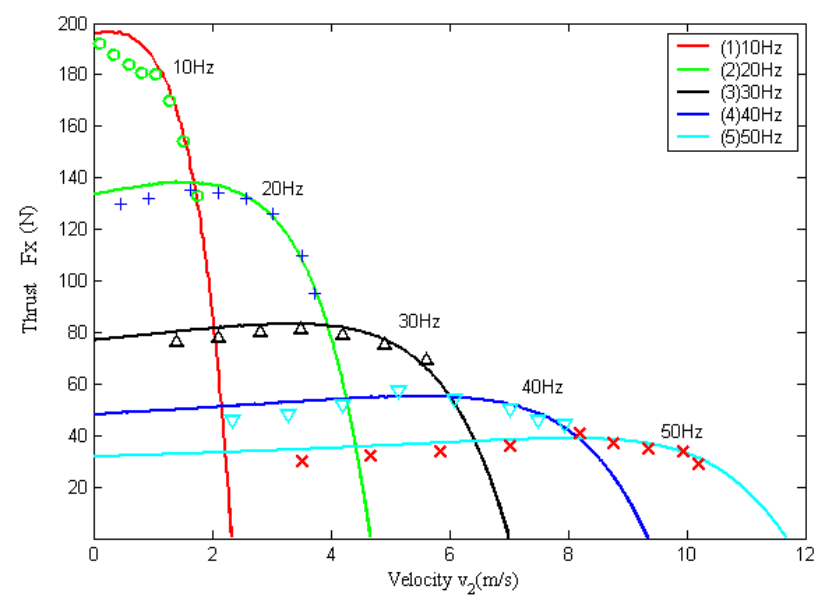

(b)

Fig. 8. Steady thrusts of the SLIM prototype: (a) Constant current constant frequency mode (solid lines indicate the thrusts without end effect, dashed lines the thrusts with end effects, others the measured thrusts); (b) Constant voltage constant frequency mode (solid lines show the thrust with end effect, others the measured thrusts).

\section{TwO-AXIS EQUiVALENT CIRCUITS}

According to the Park coordinate transformation by the power conversion rule, we can obtain the two axis $(d q$ or $\alpha \beta)$ equivalent circuits. The flux linkage matrix is

$$
\left[\begin{array}{l}
\psi_{d s} \\
\psi_{q s} \\
\psi_{d r} \\
\psi_{q r}
\end{array}\right]=
$$

$$
\left[\begin{array}{cccc}
K_{x} C_{x} L_{m}+L_{l s} & 0 & K_{x} C_{x} L_{m} & 0 \\
0 & K_{x} C_{x} L_{m}+L_{l s} & 0 & K_{x} C_{x} L_{m} \\
K_{x} C_{x} L_{m} & 0 & K_{x} C_{x} L_{m}+L_{l r} & 0 \\
0 & K_{x} C_{x} L_{m} & 0 & K_{x} C_{x} L_{m}+L_{l r}
\end{array}\right]\left[\begin{array}{l}
i_{d s} \\
i_{q s} \\
i_{d r} \\
i_{q r}
\end{array}\right]
$$

where $L_{\mathrm{m}}$ is the equivalent mutual inductance in the two-axis frame, which is equal to $1.5 L_{\mathrm{m} 1}$. In order to simplify the flux matrix expression, the symbol $s$ in the four efficiencies is omitted. We define that $L_{m c}=K_{x} C_{x} L_{m}, L_{s}=K_{x} C_{x} L_{m}+L_{l s}$, $L_{r}=K_{x} C_{x} L_{m}+L_{l r}$, then the four flux linkage expressions are further expressed by

$$
\left\{\begin{array}{l}
\psi_{d s}=L_{s} i_{d s}+L_{m c} i_{d r} \\
\psi_{q s}=L_{s} i_{q s}+L_{m c} i_{q r} \\
\psi_{d r}=L_{m c} i_{d s}+L_{r} i_{d r} \\
\psi_{q r}=L_{m c} i_{q s}+L_{r} i_{q r}
\end{array}\right.
$$

From (18), it can be seen that the four SLIM flux linkage equations are totally similar to those of the RIM. The special performance traits resulted from the longitudinal end effect, transversal edge effect, and half-filled slots are easily described by the four efficient and equivalent pole pairs. The SLIM analysis procedure and algorithm in the two-axis coordination are also similar with those of the RIM. Fig. 9 indicates the SLIM equivalent circuits in the $d q$-axis frame. Different from those of the RIM, four more rectification coefficients, i.e. $K_{\mathrm{x}}, C_{\mathrm{x}}, K_{\mathrm{r}}, C_{\mathrm{r}}$, are used in the mutual inductance and secondary resistance branch circuits. In Fig. 9, $\omega_{11}$ and $\omega_{12}$ are the angular frequencies of the primary and secondary relative to $d q$-axis.

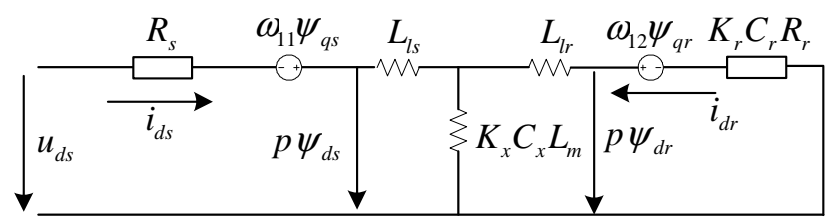

(a)

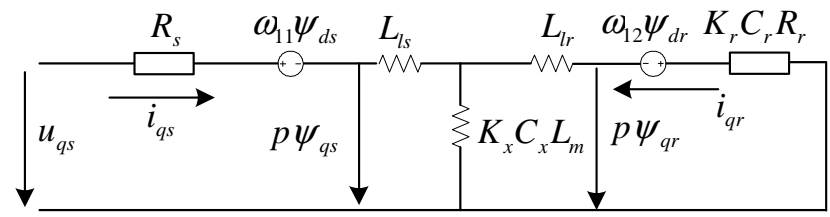

(b)

Fig. 9. Equivalent circuits of SLIM in $d q$-axis coordination: (a) $d$-axis circuit; (b) $q$-axis circuit.

\section{ANALYSIS ON FOUR COEFFICIENTS}

T-model circuit parameters for the 3-phase arc SLIM experimental prototype are: $R_{\mathrm{s}}=0.425 \Omega, L_{l s}=2.145 \mathrm{mH}$, $L_{m 1}=7.670 \mathrm{mH}, R_{r}=0.221 \Omega$, and $L_{l r}=0.550 \mathrm{mH}$. Despite the nature of the topology of the cut-open SLIM, it is found that the equivalent circuits across all the three phases are approximately equal. In order to investigate the end effects on the mutual inductance and secondary resistance conveniently, we can analyze different variable curves in the arc SLIM start-up procedure.

The primary input frequency varies from $1 \mathrm{~Hz}$ to $30 \mathrm{~Hz}$. For each input frequency the phase current changes from 10 to $30 \mathrm{~A}$. The four-coefficient curves in different frequencies are shown in Figs. 10 to 13. Figs. 14 and 15 show the curves for the mutual inductance and secondary resistance as the excited frequency goes up. The thrust along with phase current and excited frequency is given in Fig. 16. Because of end effects, the average air-gap flux density decreases with the growing velocity, which reduces the mutual inductance and increases the secondary resistance [16].

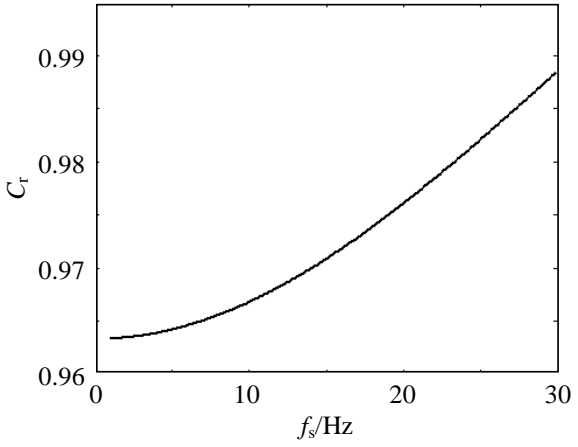

Fig. 10. Curve of coefficient $C_{\mathrm{r}}$ versus slip frequency 


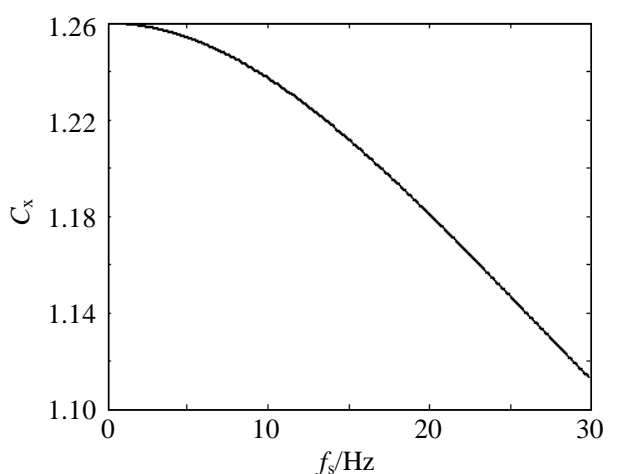

Fig. 11. Curve of coefficient $C_{\mathrm{x}}$ versus slip frequency

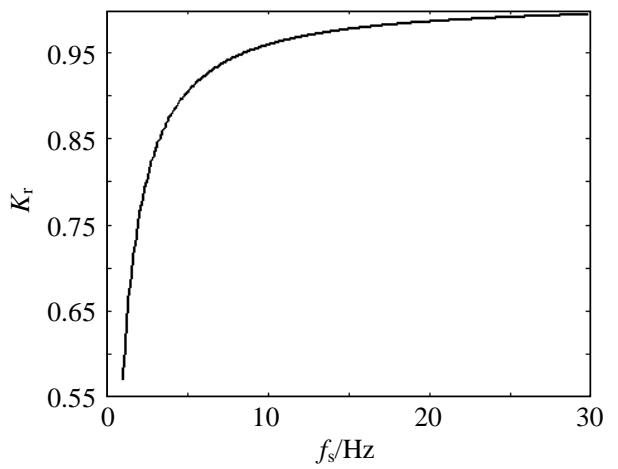

Fig. 12. Curve of coefficient $K_{\mathrm{r}}$ versus slip frequency

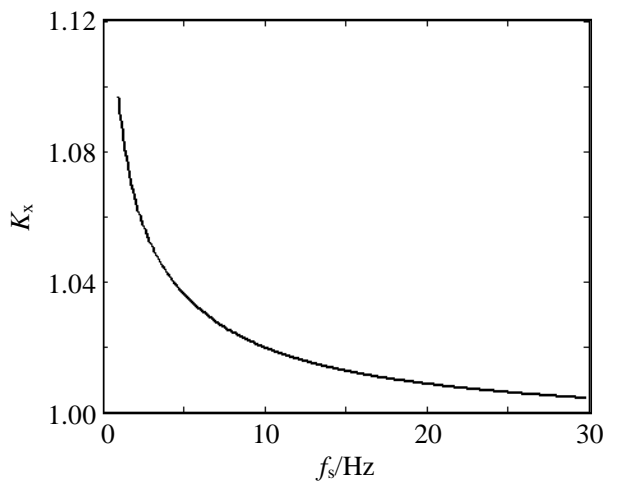

Fig. 13. Curve of coefficient $K_{\mathrm{x}}$ versus slip frequency

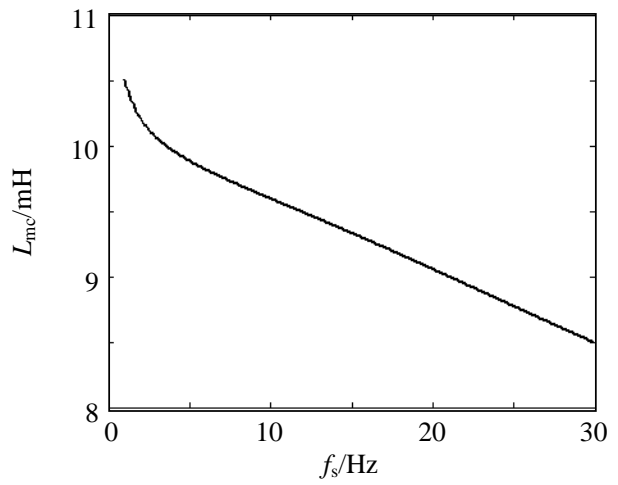

Fig. 14. Curve of modified mutual inductance $L_{m c}$ versus slip frequency

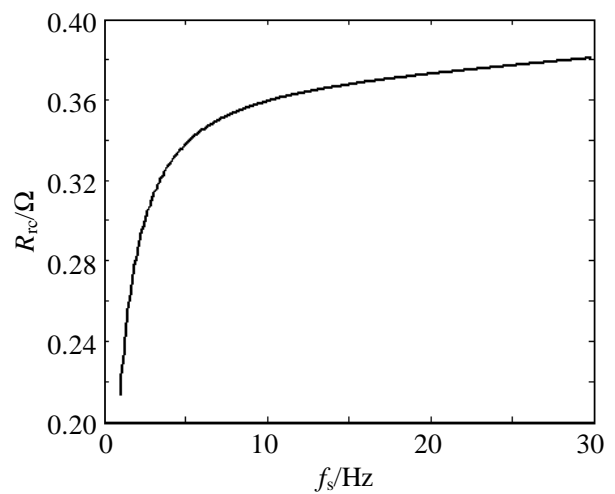

Fig. 15. Curve of modified secondary resistance $R_{\mathrm{rc}}$ versus slip frequency

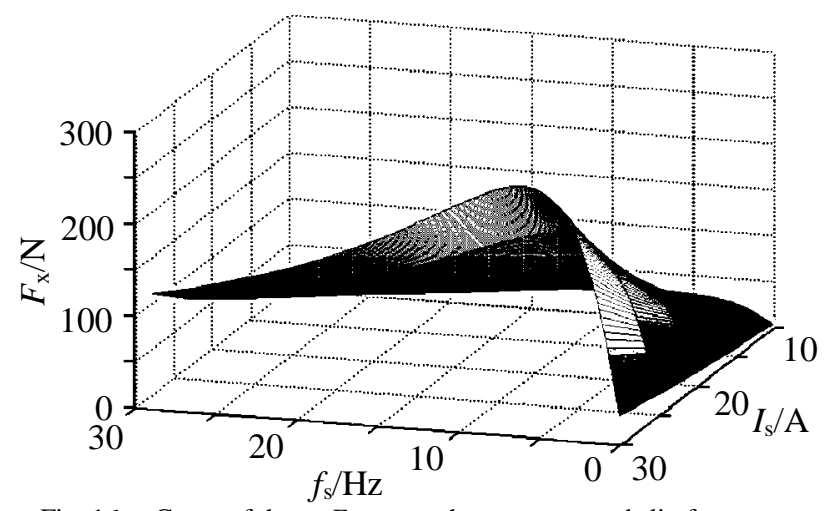

Fig. 16. Curve of thrust $F_{x}$ versus phase current and slip frequency

\section{EXPERIMENT IN INDIRECT ORIENTATION CONTROL SCHEME}

Fig. 17 shows the indirect rotor field control scheme which is similar to an RIM control. There are three closed loops, including the speed, $d$-axis current and $q$-axis current loops, which are modified by three PI regulators [16]. Fig. 18 shows the simulation and experimental curves of thrust and velocity, which agree well with each other.

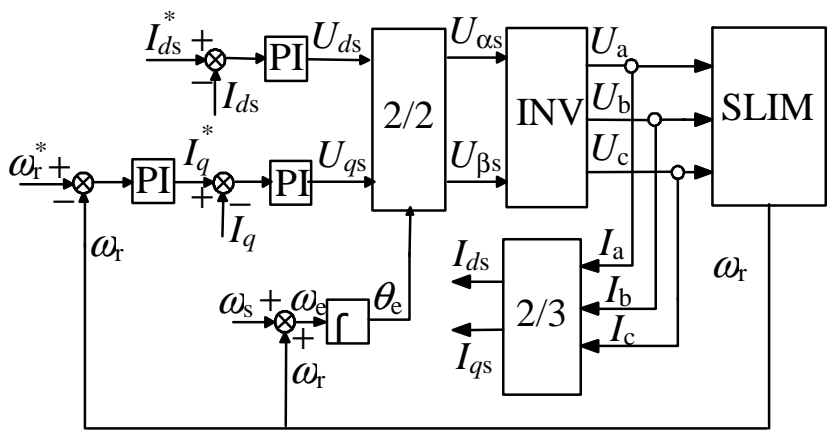

Fig. 17. Indirect orientation control analysis diagram 


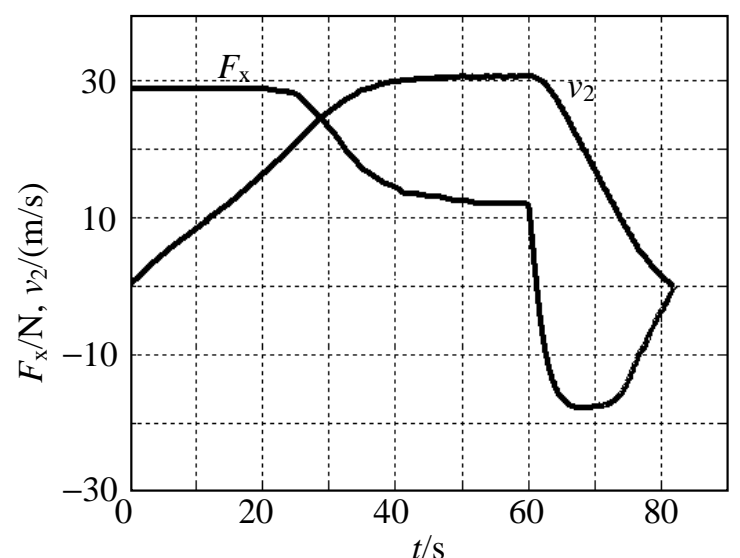

(a)

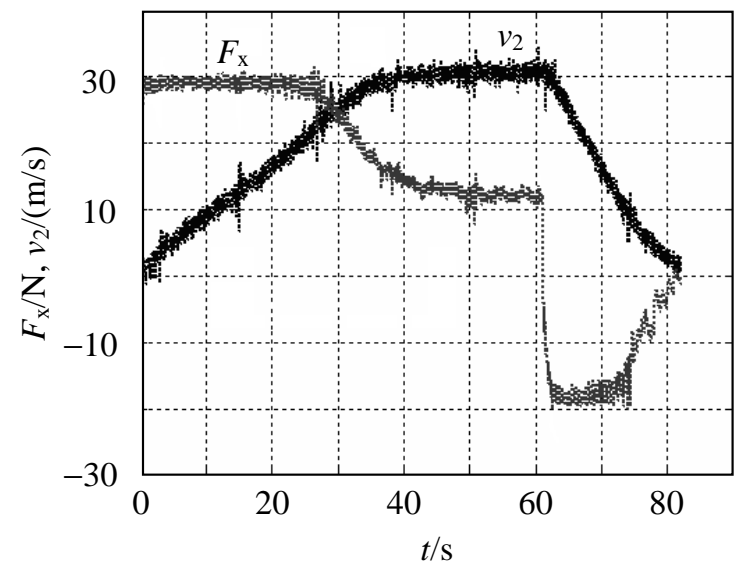

(b)

Fig. 18. Thrust and velocity curves (the ratio of thrust or velocity is 1:5 or 5:1): (a) Simulation; (b) Experiment.

\section{CONCLUSIONS}

A T-model equivalent circuit for the SLIM is developed in this paper which takes the longitudinal end effect, transversal edge effect, and half-filled slots into consideration. The four coefficients $K_{\mathrm{r}}(\mathrm{s}), K_{\mathrm{x}}(\mathrm{s}), C_{\mathrm{r}}(\mathrm{s})$, and $C_{\mathrm{x}}(\mathrm{s})$ and equivalent pole pairs $p_{\mathrm{e}}$ can describe the SLIM performance reasonably for the calculation on thrust, power factor, efficiency, and phase current in different working conditions. Moreover, the two-axis equivalent circuits $(d q$ or $\alpha \beta$ ) have been derived based on the T-model circuit, which are similar to those of the RIM except four coefficients used in the mutual inductance and secondary equivalent resistance. The curves for the four coefficients, mutual inductance, secondary resistance, and thrust in different velocity different phase current are given out. Moreover, the thrust and velocity in indirect orientation control scheme based on two-axis equivalent circuits are studied. The simulations show good correlation with the experiments. Further experimental analysis of the four efficiencies, mutual inductance, and secondary resistance will be put forward in the next step research.

\section{ACKNOWLEDGMENT}

The authors sincerely thank Prof. Yaohua Li and Dr. Jinqi Ren in the Institute of Electrical Engineering, Chinese Academy of Sciences, for their great suggestions on the research work of this paper.

\section{REFERENCES}

[1] J. Duncan, "Linear induction motor equivalent circuit model, " IEE Proc., vol. 130, Part B, no. 1, pp. 51-57, Jan. 1983.

[2] Jeong-Hyoun Sung, and Kwanghee Nam, "A new approach to vector control for a linear induction motor considering end effects," in Proc. IEEE IAS Annual Meeting, 1999, pp. 2284-2289.

[3] G. Kang, and K. Nam, "Field-oriented control scheme for linear induction motor with the end effect," IEE Proceeding on Electrical Power Application, vol. 152, no. 6, pp. 1565-1572, Nov. 2005.

[4] T. A. Lipo, and T. A. Nondahl, "Pole by pole d-q model of a linear induction machine," IEEE Trans. Power Apparatus and Systems, vol. PAS-98, no. 2, pp. 629-642, Mar./Apr. 1979.

[5] Changan Lu, "A new coupled-circuit model of a linear induction motor and its application to steady-state, transient, dynamic and control studies," Ph.D. Dissertation, Department of Electrical Engineering, Queen University, Canada, 1993.

[6] K. Idir, G. E. Dawson, and A. R. Eastham, "Modeling and performance of linear induction motor with saturable primary," IEEE Trans. Ind. Appl., vol. 29, no. 6, pp. 1123-1128, Nov./Dec. 1993.

[7] J. F. Gieras, G. E. Dawson, and A. R. Eastham, "A new longitudinal end effect factor for linear induction motors," IEEE. Trans. Energy Conversion, vol. EC-2, no. 1, pp. 152-159, Mar. 1987.

[8] J. Faiz and H. Jafari, "Accurate modeling of single-sided linear induction motor considers end effect and equivalent thickness," IEEE. Trans. Magn., vol. 36, no. 5, pp. 3785-3790, Sept. 2000.

[9] Yoshida, K., "New transfer-matrix theory of linear induction machines, taking into account longitudinal and transverse ferromagnetic end effects”, IEE Proc. Elect. Power Appl., vol. 128, no. 5, pp. 225-236, Sept. 1981.

[10] Y. Mori, S. Torii, and D. Ebihara, "End effect analysis of linear induction motor based on the wavelet transform technique," IEEE. Trans. Magn., vol. 35, no. 5, pp. 3739-3741, Sept. 1999.

[11] Tsuyoshi Higuchi and Sakutaro Nonaka, "On the design of high efficiency linear induction motors for linear metro," Electrical Engineering in Japan, vol. 137, no. 2, pp. 36-43, Aug. 2001.

[12] Xialing Long, Theory and Magnetic Design Method of Linear Induction Motor, China: Science Publishing Company, pp. 50-150, 2006.

[13] Wei Xu, "Research on the performance of single-sided linear induction motor," Ph.D. Dissertation, Institute of Electrical Engineering, Chinese Academy of Sciences, China, 2008.

[14] Wei Xu, Yaohua Li, Guangsheng Sun, and Jinqi Ren, "Performance study on high power linear induction motor in transportation," in Proc. Int. Conf. Electrical Machines and Systems, Oct. 2007, pp.1025-1027.

[15] Wei Xu, Jianguo Zhu, Longcheng Tan, Youguang Guo, Shuhong Wang, and Yi Wang, "Optimal design of a linear induction motor applied in transportation," in Proc. IEEE Int. Conf. Industry Technology, Feb. 2009, pp. 791-795.

[16] Wei Xu, Yaohua Li, Guangsheng Sun, and Jinqi Ren, "Research on new equivalent circuits of single side linear induction motor," Proceedings of the China Society for Electrical Engineering, vol. 29, no. 9, pp. 80-86, Mar. 2009. 